\title{
Influence Analysis of Reinforcement in outer Frame on the Mechanical Behavior of Multi-ribbed Frame Grid
}

\author{
Quan Yuan ${ }^{1}$, Haitao Liu ${ }^{1, *}$ Xunliu Wang ${ }^{2}$ and Yuanyuan Zhao ${ }^{1}$ \\ ${ }^{1}$ School of Civil Engineering, Beijing Jiaotong University, Beijing 100044, China \\ ${ }^{2}$ China Electronics Engineering Design Institute, Beijing 100840, China
}

\begin{abstract}
Multi-ribbed frame grid (hereinafter referred to as MRFG) is an important part of Multi-ribbed composite wall and consisted of outer frame and ribbed grid. The reinforcement of outer frame is the main influence factor on the performance of MRFG. In this study, an MRFG specimen under laterally cyclic and axial loading in literature is numerically simulated with 5 kinds of different reinforcement ratio of outer frame, by a general finite element software and user-defined material model. The influence of different reinforcement ratio of outer frame on the mechanical behavior of MRFG has been discussed.
\end{abstract}

Keywords: Multi-ribbed frame grid (MRFG), multi-ribbed composite wall, mechanical behavior, numerical simulation.

\section{INTRODUCTION}

Multi-ribbed frame grid (hereinafter referred to as MRFG), the important part of Multi-ribbed composite wall, is consisted of outer frame and ribbed grid. As the important foundation of research on multi-ribbed composite wall structure, which is a kind of low-carbon and anti-seismic structure system, the mechanical performance of MRFG needs to be thoroughly studied. Due to the complex of material properties and mechanic performance, the systematic research on mechanical behavior of MRFG under cyclic loading, such as earthquake, depends not only on model test but also on numerical simulation. The existing researches [1-3] have showed that the reinforcement of outer frame is the main influence factor on the mechanical performance of MRFG. In this study, by a general finite element software and user-defined material model, an MRFG specimen under laterally cyclic and axial loading in literature [4] is numerically simulated with 5 kinds of different reinforcement ratio of outer frame. The influence of different reinforcement ratio of outer frame on the mechanical behavior of MRFG has been discussed.

\section{FINITE ELEMENT MODELING AND ANALYZING}

\subsection{Material Model}

In this study, the "WANG steel model" [5-9], which has been verified by test, is used as constitutive model of steel/strand. This model can describe the common steel with yielding point or hard steel/strand without yielding point, in which Bauschinger effect is reasonably considered. Monotonic loading envelope of steel/strand material model adopts Esmaeily-Xiao curve, unloading curve uses linear curve and

*Address correspondence to this author at the School of Civil Engineering, Beijing Jiaotong University, Beijing 100044, China; Tel: +86-10-51687249; E-mails: 07115260@bjtu.edu.cn, lhait2011@sina.com reloading envelope adopts Légeron curve. "WANG steel model" is showed in Fig. (1), the meanings of parameters in which can be found in literature [5].

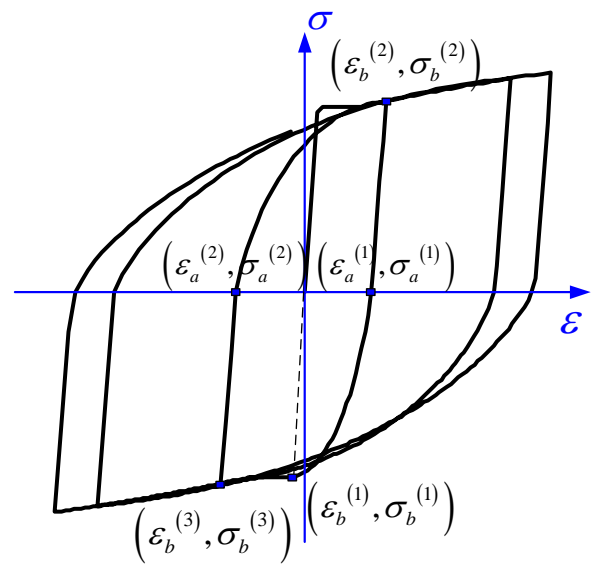

Fig. (1). The constitutive model of steel (i.e. WANG steel model).

Based on types of the elements in use, the "WANG concrete model" $[5,6]$ or "classical concrete elastic-plastic + fracture constitutive model" is utilized to describe the constitutive model of concrete ado. "WANG concrete model" is an uniaxial constitutive model, in which the accumulative damage is considered, and it is mainly used in fiber beam-column element. In this model, the monotonic compressive loading envelope adopts Légeron-Paultre curve, monotonic tensile loading envelope uses JIANG Jianjing curve, loading-unloading constitutive model considers stiffness degradation and hysteretic characteristics and a transition region between tension and compression is established, as showed in Fig. (2). What's more, the "WANG concrete model" is able to judge whether the concrete to be modeled is confined or not according to conditions of reinforcement by itself and calculate the parameter 
$\mathrm{I}_{\mathrm{e} 0}$ automatically. The "classical concrete elastic-plastic + fracture constitutive model" is used in multi-layer shell.

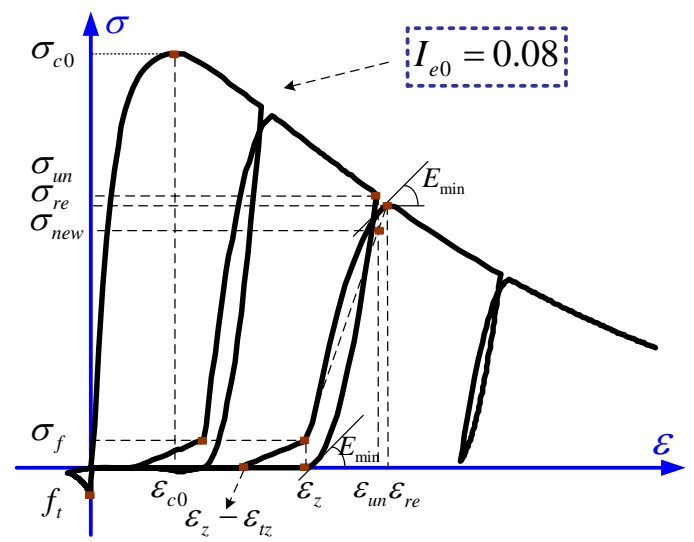

Fig. (2). The constitutive model of concrete (i.e. WANG concrete model).

\subsection{Finite Element Modeling}

Finite element modeling should be in accordance with actual boundary condition of the specimen. The failure process of specimen 5 under laterally cyclic and axial loading in literature 3 is described as follows. Concrete crushing and reinforcement yielding appear in rib-beam firstly, then in outer beam and rib-column, and in out column lastly. In the limit state, one outer column fails in tension, and the other fails in compression, exhibiting a failure mode of shear wall. In the finite element modeling, it can be considered that inner rib-grid bears mainly bending deformation, while outer rib and outer frame bear mainly shear deformation for the supporting function. So, inner rib-grid is discretized by fiber beam-column elements, which use "WANG steel model" (showed in Fig. 1) and "WANG concrete model" (showed in Fig. 2) as constitutive model. Outer rib and outer frame is discretized by multi-layer shell elements, in which the inplane shear deformation can be taken into account and the "classical concrete elastic-plastic + fracture constitutive model" and the "WANG steel mode" are used as material constitutive models. It is noteworthy that the bar element utilizes the function of "INSERT" to realize the connection of steel and concrete. Finite element modeling is showed in Fig. (3).

\subsection{Numerical Calculation and Results}

Based on the method of finite element modeling above, MRFG specimen 5 under laterally cyclic and axial loading in literature [3] is simulated numerically with a maximal displacement angle of $1 / 30$. The section dimension, reinforcement, material parameters and loading method can be found in literature [3]. In the numerical calculation, strict convergence criteria of force and displacement (3\%) are used. In the part of fiber beam-column element, the section of member is divided into $6 \times 6$ concrete fibers and bar fibers which has the same number as longitudinal steels, and in the part of multi-layer shell element, the concrete shell is divided 10 layers along thickness direction which also has the same number of bar as longitudinal steels. From the comparison of testing and calculating, showed in Fig. (4), it can be found that their bearing capacities are approximately equal and their hysteretic curves are basically coincidence.

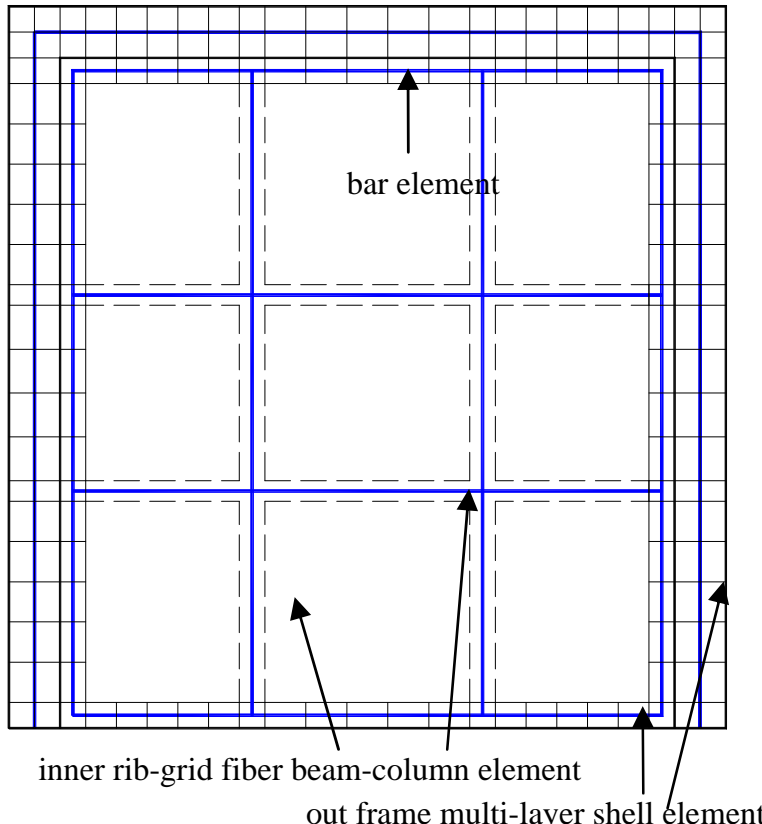

Fig. (3). Finite element modeling.

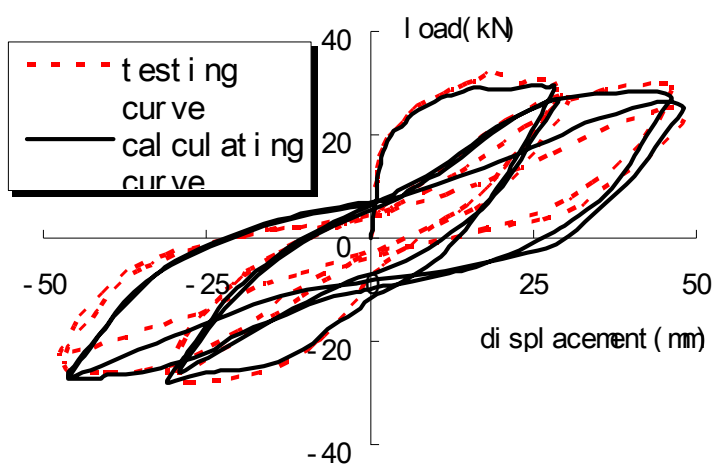

Fig. (4). The comparison of testing and calculating.

\section{INFLUENCE ANALYSIS OF REINFORCEMENT IN OUTER FRAME OF MRFG}

As showed in literature [3], there are many factors effecting the mechanical behavior of MRFG, such as the section dimensions and reinforcement of outer frame, the section dimensions and reinforcement of ribbed grid, concrete strength, etc, in which the reinforcement of outer frame may have the greatest influence. In order to analyze the effect to the reinforcement of outer frame on bearing capacity, stiffness and energy dissipation of MRFG, a specimen is chosen from actual structure, which section dimension and material parameter can be found in the specimen SW-1 of the literature [4]. The specimen is simulated numerically by using above method, which has been test verified. In the simulation, there are two kinds of loading modes, one is monotonic loading (the maximum of displacement is $100 \mathrm{~mm}$ ) for evaluating the bearing capacity and stiffness, and the other is cyclic loading (the maximum of displacement angle is 1/50) for assessing the energy dissipation. The results are showed in Figs. 5-9 and Tables 1, 2. 


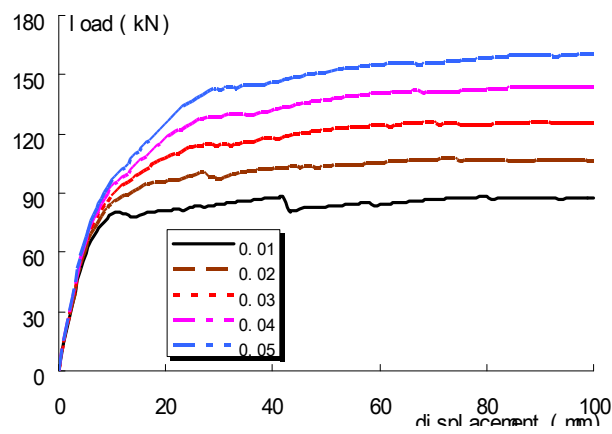

(a) Result under monotonic loading

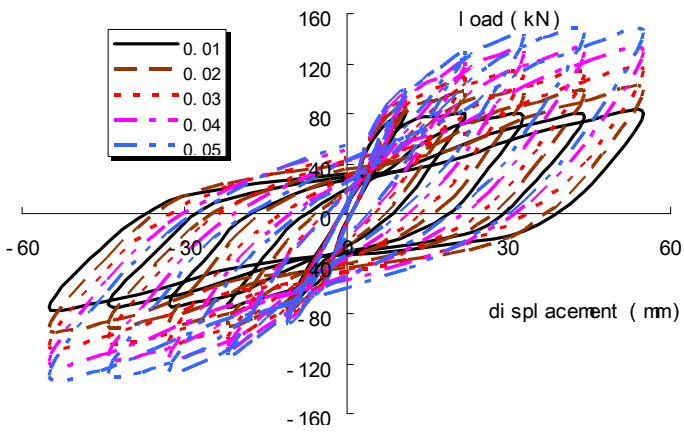

(b) Result under cyclic loading

Fig. (5). The result of numerical calculation under various reinforcement ratio of outer frame columns.

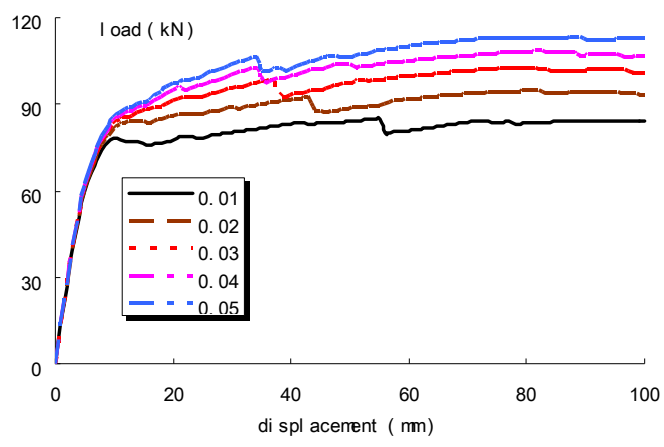

(a) Result under monotonic loading

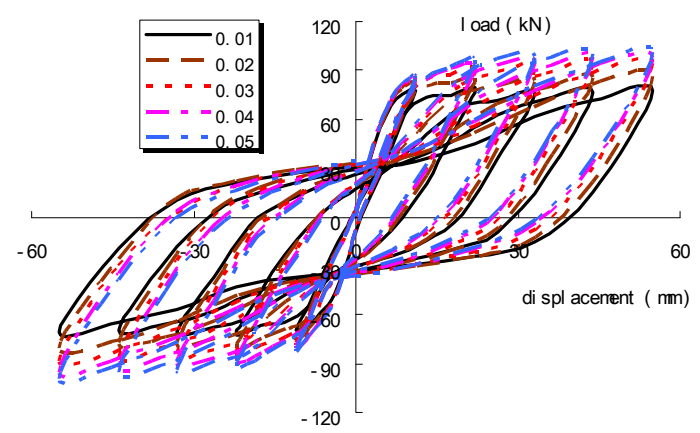

(b) Result under cyclic loading

Fig. (6). The result of numerical calculation under various reinforcement ratio of outer frame beams.

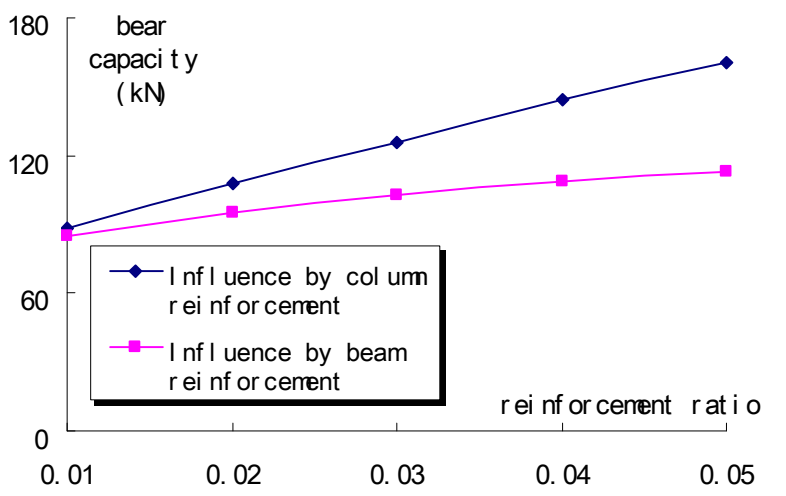

Fig. (7). The relationship between Bear Capacity and Reinforcement Ratio.

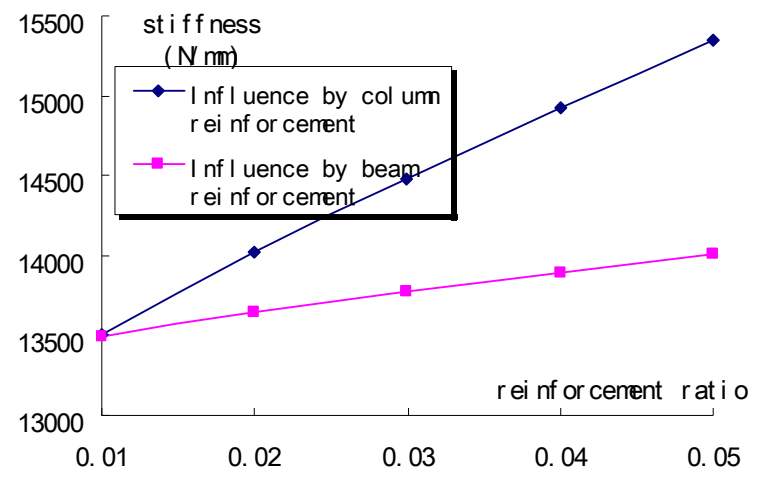

Fig. (8). The relationship between Stiffness and Reinforcement Ratio.

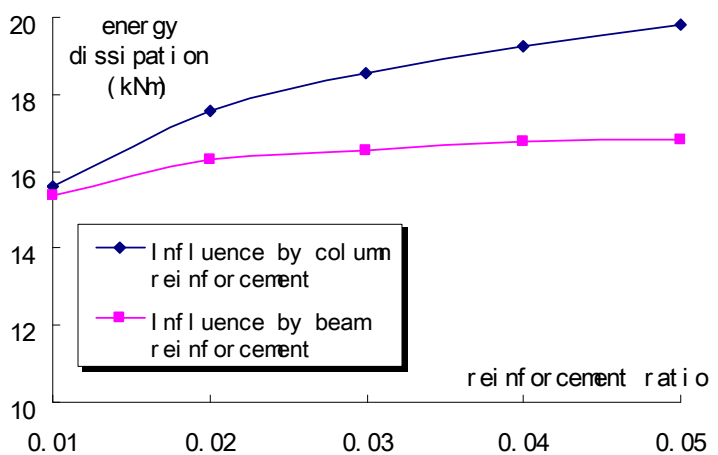

Fig. (9). The relationship between Energy Dissipation and Reinforcement Ratio.

\subsection{Analysis of Influence on Reinforcement of Outer Frame Columns}

As showed in Fig. 5-9 and Table 1, the variation of reinforcement in outer frame columns has great influence on the mechanical behavior of MRFG. In the range of normal reinforcement ratio (between $1 \%$ and $5 \%$ ), the bearing capacity, stiffness and energy dissipation of MRFG are almost proportional to the reinforcement ratio. When increasing the reinforcement ratio of outer frame columns from $1 \%$ to $5 \%$, the bearing capacity, stiffness and energy dissipation is added $82.1 \%, 13.6 \%$ and $27 \%$, respectively. Comparatively speaking, the greatest influence is on bearing 
Table 1. The Result of Bear Capacity, Stiffness and Energy Dissipation Under Various Reinforcement Ratio of Outer Frame Columns

\begin{tabular}{|c|c|c|c|c|c|}
\hline & Case 1 & Case 2 & Case 3 & Case 4 & Case 5 \\
\hline reinforcement ratio & $1 \%$ & $2 \%$ & $3 \%$ & $4 \%$ & $5 \%$ \\
\hline bear capacity $(\mathrm{N})$ & 88229 & 107595 & 125924 & 144182 & 160700 \\
\hline stiffness (N/mm) & 13503.5 & 14018.8 & 14478.4 & 14923.6 & 15346.7 \\
\hline energy dissipation $(\mathrm{N} \cdot \mathrm{m})$ & 15589568 & 17590021 & 18567827 & 19230351 & 19798459 \\
\hline
\end{tabular}

Table 2. The resuçlt of Bear Capacity, Stiffness and Energy Dissipation Under Various Reinforcement Ratio of Outer Frame Beams

\begin{tabular}{|c|c|c|c|c|c|}
\hline & Case 1 & Case 2 & Case 3 & Case 4 & Case 5 \\
\hline reinforcement ratio & $1 \%$ & $2 \%$ & $3 \%$ & $4 \%$ & $5 \%$ \\
\hline bear capacity $(\mathrm{N})$ & 84595.1 & 94817.3 & 102702 & 108382 & 113211 \\
\hline stiffness (N/mm) & 13495.84 & 13646.65 & 13769.53 & 13891.97 & 14003.65 \\
\hline energy dissipation $(\mathrm{N} \cdot \mathrm{m})$ & 15365338 & 16291513 & 16548725 & 16764326 & 16834074 \\
\hline
\end{tabular}

capacity, the next is on energy dissipation, and the least is on stiffness. From the relationship curve between energy dissipation and reinforcement ratio (as showed in Fig. 9), it can be found that the energy dissipation increases faster with relatively small reinforcement ratio, for instance, the increment is $12.8 \%$ from reinforcement ratio of $1 \%$ to $2 \%$, but the increment is only $4.2 \%$ from ratio of $4 \%$ to $5 \%$.

\subsection{Analysis of Influence on Reinforcement of Outer Frame Beams}

As showed in Figs. 6-9 and Table 2, the variation of reinforcement in outer frame beams also has some influence on the mechanical behavior of MRFG. In the range of normal reinforcement ratio (total ratio of section reinforcement is between $1 \%$ and $5 \%$, namely, the ratio of tensile steel is between $0.5 \%$ and $2.5 \%$ ), the bearing capacity, stiffness and energy dissipation of MRFG are also almost proportional to the reinforcement ratio. When increasing the reinforcement ratio of outer frame beams from $1 \%$ to $5 \%$, the bearing capacity, stiffness and energy dissipation is added $33.8 \%, 3.8 \%$ and $9.6 \%$, respectively. As the influence trends of outer frame columns reinforcement, the greatest influence by outer frame beams reinforcement is on bearing capacity, the next is on energy dissipation, and the least is on stiffness. From the relationship curve between energy dissipation and reinforcement ratio (as showed in Fig. 9), it can be found that the energy dissipation increases faster with relatively small reinforcement ratio, for instance, the increment is $6.0 \%$ from reinforcement ratio of $1 \%$ to $2 \%$, but the increment is only $1.1 \%$ from ratio of $4 \%$ to $5 \%$.

\section{CONCLUSION}

(1) By the numerical simulation of MRFG specimen [3] under cyclic loading, it can draw a conclusion: under complex stress condition, MRFG specimen shows the stress and deformation behavior of shear wall. So the modeling method of frame is not suitable, while the method of inner-beam and outer-shell has high precision compared with the test result.

(2) The reinforcement in outer frame may have a great influence on the mechanical behavior of MRFG. In the range of normal reinforcement ratio, the bearing capacity, stiffness and energy dissipation of MRFG are almost proportional to the reinforcement ratio. Among them, the greatest influence is on bearing capacity, the next is on energy dissipation, and the least is on stiffness.

(3) From energy dissipation, it can be found that the energy dissipation increases faster with relatively small reinforcement ratio, while become slower with high ratio.

(4) Comparatively speaking, the reinforcement of outer frame columns has greater influence on mechanical behavior of multi-ribbed frame grid than the reinforcement of outer frame beams.

\section{ACKNOWLEDGMENTS}

The authors of this paper acknowledge the support of the National Natural Science Foundation of China (Grant No. 50878021, 50908188), the Key Projects in the National Science \& Technology Pillar Program during the Eleventh Five-Year Plan Period (Grant No. 2006BAJ04A02-05).

\section{REFERENCES}

[1] Q. F. Yao, and Y. Zhang, "Development and application of new architectural structure system of residence", Industrial Construction, vol. 32, pp. 53-56, August 2002.

[2] W. Huang, and Q. F. Yao, "Analyses of seismic performance of multi-ribbed wall slab with light-weight outer frame", Industrial Construction, vol. 33, pp. 6-9, January 2002. 
[3] J. Zhang, "Mechanical Property Study and Practical Oblique Section Design Method Study on Multi-ribbed Composite Wall", M.S. thesis, Xi'an University of Architecture and Technology, Xi'an, China, 2004.

[4] W. Huang, "Research of Seismic Performance and Design Theory of Multi-ribbed Slab Wall", Ph.D. thesis, Xi'an University of Architecture and Technology, Xi' an, China, 2004.

[5] X. L. Wang, "Research on Re-centering Behavior of Reinforced Concrete Columns with Unbonded High-Strength Strands", Ph.D. thesis, Tsinghua University, Beijing, China, 2007.

[6] X. Z. Lu, L. P. Ye, and Z. W. Miao, Elasto-plastic Analysis of Buildings against Earthquake-Theory, Model and Implementation on ABAOUS, MSC.MARC and SAP2000, Beijing: China Architecture \& Building Press, 2009.

[7] Z. W. Miao, X. Z. Lu, and L. P. Ye, "Application of microplane models in the computation of shear wall structure", Journal of Shenzhen University (Science \&engineering), vol. 25, pp. 122-128, February 2008.

[8] X. L. Wang, X. Z. Lu, and L. P. Ye, "Numerical simulation for the hysteresis behavior of rc columns under cyclic loads", Engineering Mechanics, vol. 24, pp. 76-81, December 2007.

[9] X. C. Lin, X. Z. Lu, and Z. W. Miao, "Finite element analysis and engineering application of rc core-tube structures based on the multi-layer shell elements", China Civil Engineering Journal, vol. 42, pp. 51-56, March 2009.

(c) Yuan et al.; Licensee Bentham Open.

This is an open access article licensed under the terms of the Creative Commons Attribution Non-Commercial License

(http://creativecommons.org/licenses/_by-nc/3.0/) which permits unrestricted, non-commercial use, distribution and reproduction in any medium, provided the work is properly cited. 